\title{
COMO A CORÉIA DE SYDENHAM É TRATADA NO RIO DE JANEIRO?
}

\author{
Vinicius Castro Souza', Alexandra Prufer Araújo², Charles André ${ }^{3}$
}

\begin{abstract}
RESUMO - Introdução: A coréia de Sydenham é a principal causa de coréia adquirida na infância no Brasil. Assim, torna-se relevante saber como os médicos tratam os pacientes coréicos no nosso meio. Objetivo: Descrever a prática médica informada em coréia de Sydenham entre pediatras. Método: Estudo observacional descritivo seccional realizado por entrevistas feitas entre pediatras de emergência e especialistas dos hospitais públicos do Município do Rio de Janeiro. Resultados: $74 \%$ dos entrevistados referiu não usar escalas de gravidade; somente $81 \%$ dos médicos fazem uso regular de penicilina benzatina; $64 \%$ referem iniciar tratamento farmacológico; $28,3 \%$ usam apenas o haloperidol para o tratamento. Conclusão: As escalas de gravidade não são usadas rotineiramente no atendimento de pacientes coréicos; há tendência à prescrição irregular de penicilina entre médicos mais jovens; o haloperidol é a droga mais prescrita entre os entrevistados.
\end{abstract}

PALAVRAS-CHAVE: transtornos coréicos, febre reumática, condutas na prática dos médicos.

\begin{abstract}
How Sydenham's chorea is treated in Rio de Janeiro?
ABSTRACT - Background: Sydenham's chorea is the most common cause of acquired childhood chorea in Brazil. Thus it is relevant to know how physicians treat those patients. Objective: To describe the practice patterns of Sydenham's chorea among pediatricians. Method: A descriptive study was undertaken using a questionnaire among specialists and emergency pediatricians who work in public hospitals of Rio de Janeiro district. Results: $74 \%$ of the physicians informed not to use any severity scale; $81 \%$ informed to always prescribe benzatine penicillin; $64 \%$ informed to begin pharmacological treatment for all patients; and Haloperidol was the most remembered drug among all physicians. Conclusion: Physicians do not use routinely severity scales in follow-up of choreic patients; there is a tendency of irregular prescription of benzatine penicillin by younger doctors; and $28.3 \%$ prescribe only haloperidol.
\end{abstract}

KEY WORDS: choreatic disorders, rheumatic fever, physician's practice patterns.

A coréia de Sydenham (CS) é a principal manifestação neurológica da febre reumática (FR): geralmente afeta crianças de 5 a 15 anos e é um critério maior para o diagnóstico de $\mathrm{FR}^{1-3}$. Caracteriza-se por movimentos imprevisíveis, contínuos, porém rápidos, irregulares e breves envolvendo diferentes grupos musculares $^{4,5}$. Apesar de ter sua incidência reduzida nos últimos anos, principalmente em países desenvolvidos, ainda constitui, no Brasil, a principal causa de coréia adquirida na infância ${ }^{6,7}$. A incidência de CS em países desenvolvidos é de 0,2 a 0,8 por 100000 por ano, o que representa 10 a $40 \%$ dos pacientes com $\mathrm{FR}^{3,8-11}$. A taxa de recorrência é de 10 a $42 \%^{10-12}$. Cerca de 60 a $71 \%$ dos pacientes com CS apresentam lesões cardíacas valvares ${ }^{3,10,13}$. Esta última é a principal responsável pela morbidade crônica e mortalidade dos pacientes ${ }^{2,4,14}$. A fisiopatologia da CS tor- nou-se mais conhecida nos últimos anos. Uma das teorias mais aceitas é a de que os anticorpos produzidos pelo hospedeiro para combater o estreptococo beta-hemolítico do grupo A fazem reação cruzada com proteínas de superfície de neurônios específicos dos núcleos da base, em processo que se chama de mimetização molecular ${ }^{9,11,15}$. Conseqüentemente reflete alteração em núcleos da base, seja exclusivamente neuroquímica, uma disfunção dopaminérgica, ou associada a uma alteração anatômica ${ }^{3,9,16,17}$. Porém, as alterações de humor e síndromes psiquiátricas são freqüentemente relatadas precedendo, sucedendo ou acompanhando o quadro coréico ${ }^{3,6,9,11}$. Isto sugere que a lesão causada pela resposta ao estreptococo seja direcionada aos núcleos da base, mas não restrita a eles, acometendo, portanto, o cérebro de forma mais ampla. Ademais, há autores que rea-

Instituto de Pediatria e Puericultura Martagão Gesteira (IPPMG) / Universidade Federal do Rio de Janeiro (UFRJ), Rio de Janeiro RJ, Brasil: ${ }^{1}$ Mestrando, Neuropediatra do Hospital Geral de Bonsucesso; ${ }^{2}$ Pós-Doutora, Professora Adjunta de Pediatria IPPMG/UFRJ; ${ }^{3}$ Doutor, Professor Adjunto de Neurologia UFRJ.

Recebido 16 Outubro 2006, recebido na forma final 21 Fevereiro 2007. Aceito 11 Abril 2007. 
lizaram estudos histopatológicos em casos de coréia e que mostraram alterações cerebrais mais difusas envolvendo inclusive o córtex cerebral ${ }^{18}$. 0 diagnóstico é clínico, afastando-se outras causas de coréia; não há exames laboratoriais para confirmação. $O$ início da coréia ocorre, geralmente, de 1 a 6 meses depois da infecção estreptocócica ${ }^{2,19}$; portanto, os testes para se identificar infecção estreptocócica raramente são positivos ${ }^{9,11}$. A gravidade é variável e foi criada uma escala para acompanhamento de sua evolução ${ }^{20}$. O prognóstico é bom pelo caráter auto-limitado da coréia $a^{4,6,7,8,11}$, com remissão completa dos sintomas em até 6 meses $^{3,7,8,15}$ em metade dos pacientes; em 40 a $50 \%$ dos casos, porém, pode haver persistência de coréia, de leve a moderada por mais de $2 \operatorname{anos}^{3,7,8,15,17 .}$

O tratamento farmacológico pode se subdividir em três categorias: drogas cujo mecanismo de ação principal é depletar ou antagonizar a dopamina em núcleos da base; drogas que agem principalmente aumentando os níveis de ácido gama-amino-butírico (GABA) em núcleos da base; drogas que atuam diminuindo a concentração de auto-anticorpos circulantes, reduzindo o processo inflamatório vascular nos núcleos da base. No primeiro grupo, podemos citar o haloperidol, pimozide, clorpromazina, difenilbutilpiperidina, perfenazina, carbamazepina; no segundo grupo, drogas como o ácido valpróico; no terceiro grupo encontramos os tratamentos imuno-modulatórios, como imunoglobulina, plasmaferese e prednisona ${ }^{21}$. Não há consenso sobre a terapia farmacológica para coréia. Entretanto, há unanimidade sobre o uso profilático de penicilina benzatina para evitar novos surtos de coréia ou outras manifestações da FR, principalmente a cardite ${ }^{3,11,12,22}$.

A fim de conhecer a conduta vigente nos casos de coréia reumática, diante dos avanços ocorridos, propôs-se este estudo.

\section{MÉTODO}

Trata-se de estudo observacional descritivo, baseado em questionário aplicado a pediatras que atuam em emergências da cidade do Rio de Janeiro e aos especialistas que atuam nos Centros de Referência para o atendimento da FR no município do Rio de Janeiro. A entrevista a pediatras decorre da faixa etária deste distúrbio.

Existem 8 hospitais com centros de referência para tratamento da FR no Município do Rio de Janeiro, segundo a Sociedade de Pediatria do Rio de Janeiro. São eles: Hospital da Piedade, Hospital Geral de Bonsucesso, Hospital Servidores do Estado, Hospital Municipal Jesus, Hospital Universitário Pedro Ernesto, Instituto de Pediatria e Puericultura Martagão Gesteira, Instituto Nacional de Cardiologia de Laranjeiras, Instituto Estadual de Cardiologia Aloísio de Cas- tro. Todos esses hospitais entraram no estudo. O censo foi realizado na população destes hospitais, composta por cardiopediatras, neuropediatras, reumatopediatras.

Os demais hospitais foram incluídos no estudo por meio de seleção aleatória entre os hospitais que possuem atendimento de emergência pediátrica, segundo consta no Guia de Saúde da Cidade do Rio de Janeiro, edição de 1999, distribuído pela Secretaria Municipal de Saúde do Município do Rio de Janeiro.

O objetivo principal foi descrever a prática médica informada em CS entre pediatras de emergência e especialistas dos Centros de Referência para o Tratamento da FR do Município do Rio de Janeiro, incluindo sua experiência no uso de escala de gravidade para classificação da coréia e tratamento farmacológico da coréia.

Os critérios de elegibilidade foram atuar como especialista em cardiopediatria, reumatologia ou neuropediatria nos Centros de Referência ou em emergência há mais de 6 meses e ter atendido pelo menos um caso de CS nos dois últimos anos que antecederam o estudo. Foram excluídos os médicos que não quiseram participar e os questionários preenchidos de forma incompleta.

A elaboração do questionário - As perguntas foram formuladas como resultado de uma revisão bibliográfica sobre o tema incluindo as variáveis que se desejou estudar, do modo mais claro e objetivo possível.

O questionário elaborado possui perguntas que foram organizadas em três grupos com os seguintes aspectos: perfil do entrevistado; experiência em atendimento de portadores de CS; experiência no tratamento da coréia.

O pré-teste - Após a elaboração do questionário piloto, o mesmo foi submetido aos médicos pediatras que atuam em enfermarias e ambulatório geral do Instituto de Pediatria e Puericultura Martagão Gesteira (IPPMG/UFRJ) e que não atuam simultaneamente como médicos em serviços de emergência do Município do Rio de Janeiro ou nos centros de referência para atendimento de FR, numa amostra de conveniência entre os meses de dezembro de 2003 e fevereiro de 2004.

A validade externa foi direcionada para as perguntas sobre o tipo de tratamento utilizado para pacientes exibindo coréia, uso de escala de gravidade, uso de profilaxia secundária. Foi então aferida, comparando-se as respostas dadas ao questionário pelos médicos de cada serviço do IPPMG e dados dos prontuários dos pacientes atendidos nos mesmos serviços. Ao todo foram analisados 54 prontuários referentes ao atendimento ambulatorial e de emergência de pacientes coréicos ao longo do ano de 2003.

A aplicação do questionário - O pesquisador aplicava e recolhia o questionário no mesmo momento. Não deixava, portanto, o questionário com o entrevistado para ser recoIhido em outro dia.

Os questionários foram distribuídos e recolhidos durante o período de 01/09/04 até 06/04/05.

Os dados foram digitados em banco de dados e analisados de forma descritiva com auxílio do programa Epi-Info. 
Os gráficos de freqüência foram elaborados pelo programa Microsoft Excel versão 2000. De forma exploratória, fez-se comparações entre grupos da amostra utilizando-se o teste qui-quadrado com correção de Yates para variáveis categóricas e o teste Mann-Whitney para comparação de médias.

Questões éticas - O projeto foi aprovado pelo Comitê de Ética do IPPMG em 15/06/2004.

O pesquisador preocupou-se em manter o anonimato dos médicos que foram entrevistados. Os questionários respondidos eram numerados e não assinados e só poderiam ser identificados pelo próprio entrevistado que era o único possuidor do número que os identificava.

O termo de consentimento distribuído para os médicos, com telefone e endereço do pesquisador e cópia da aprovação do comitê de ética.

\section{RESULTADOS}

Foram coletados 90 questionários, sendo 13 de hospitais de emergência e 77 de especialistas e pediatras de emergência dos centros de referência. Entre os especialistas dos centros de referência que foram entrevistados, houve perda de 6\% (Tabela 1).

Apenas $8 \%$ dos médicos referiram usar a escala de gravidade em todos os casos de coréia que atendem.

Observou-se associação entre a prescrição irregular de penicilina e o tempo de formado do médico. A freqüência de prescrição irregular de penicilina é maior entre médicos com menos de 10 anos de formados (p 0,04).

Cerca de $60 \%$ destes médicos prescrevem penicilina de forma irregular, enquanto apenas $40 \%$ dos médicos com mais de 10 anos de formados fazem prescrição irregular de profilaxia secundária.
Tabela 1. Dados descritivos de todos os entrevistados

\begin{tabular}{lc}
\hline Variáveis & Percentuais \\
\hline Uso de escala de gravidade & \\
Não, pois desconhecem & $61 \%$ \\
Sim & $26 \%$ \\
Não, apesar de conhecerem & $13 \%$ \\
Uso de penicilina benzatina & \\
Sempre & $81 \%$ \\
Só em casos graves & $16 \%$ \\
Não lembram & $2 \%$ \\
Nunca & $1 \%$ \\
Indicação de tratamento farmacológico & \\
Sempre & $64 \%$ \\
Só em casos graves & $31 \%$ \\
Nunca tratam & $4 \%$ \\
Não lembram & $1 \%$ \\
Medicação preferencial & \\
Haloperidol & \\
Corticóide & $28,9 \%$ \\
Diazepan & $2 \%$ \\
Valproato & $1 \%$ \\
Associação de drogas & $0 \%$ \\
\hline
\end{tabular}

Cerca de $64 \%$ dos entrevistados refere sempre iniciar tratamento farmacológico para seus pacientes (Tabela 2).

Os números maiores que 1 indicam maior freqüência do uso da medicação entre entrevistados dos centros de referência.

A tabela indica uma possível preferência, entre os médicos de Centros de Referência, pelo uso de corticóide para tratamento da coréia aguda, quando comparado aos pediatras de outros hospitais $(p=0,034)$.

Tabela 2. Diferenças das condutas entre entrevistados dos centros de referência e de fora dos centros de referência.

\begin{tabular}{|c|c|c|}
\hline Associação & $\begin{array}{c}\text { Razão de chances } \\
\text { (Intervalo de confiança 95\%) }\end{array}$ & $\begin{array}{l}\mathrm{p} \text {-valor } \\
\text { Yates }\end{array}$ \\
\hline Sempre faz uso de escala de gravidade & $\begin{array}{c}1,20 \\
(0,12-28,27)\end{array}$ & 0,716 \\
\hline Sempre inicia tratamento farmacológico & $\begin{array}{c}1,09 \\
(0,28-4,19)\end{array}$ & 0,868 \\
\hline Sempre usa Haloperidol na fase aguda de coréia & $\begin{array}{c}0,99 \\
\text { (Limite inválido\#) }\end{array}$ & 0,584 \\
\hline Sempre usa Valproato na fase aguda de coréia & $-*$ & 0,037 \\
\hline Sempre usa Corticóide em fase aguda de coréia & $\begin{array}{c}9 \\
(1,11-194,45)\end{array}$ & 0,034 \\
\hline Sempre usa Diazepan em fase aguda de coréia & $\begin{array}{c}0,32 \\
(0,08-1,37)\end{array}$ & 0,154 \\
\hline Sempre faz uso de Penicilina benzatina & $\begin{array}{c}0,75 \\
(0,1-4,24)\end{array}$ & 0,972 \\
\hline
\end{tabular}

"Este limite inválido deveu-se a pequena amostra de pediatras de fora dos centros de referência; *Nenhum pediatra de fora dos centros de referência citou o valproato para tratamento, logo não há intervalo de confiança e razão de chances 
Nenhum entrevistado de fora dos Centros de Referência referiu prescrever valproato para tratamento da CS.

\section{DISCUSSÃO}

Nosso estudo revelou o impressionante percentual de $16 \%$ dos entrevistados que referiu indicar penicilina apenas em certos casos de CS (Tabela 1). A revisão feita entre os 54 prontuários selecionados ratifica a hipótese de prescrição inadequada da profilaxia secundária entre os médicos, pois que em $8,7 \%$ dos prontuários pesquisados não se encontrou qualquer menção ao uso de penicilina benzatina. Uma vez que a indicação da profilaxia secundária com penicilina benzatina é amplamente recomendada na literatura médica ${ }^{3,8,12,22}$ e faz parte de virtualmente todos os livros-texto que estudam o assunto ${ }^{2,7,23}$, é com preocupação que notamos um grupo considerável de pediatras que parecem não saber da importância desta prática profilática.

Mais preocupante é encontrar este percentual em nossa amostra, porque ela foi composta em sua maioria de pediatras de centros de referência, principalmente pediatras especialistas. A alta freqüência de prescrição inadequada da profilaxia secundária para pacientes coréicos justamente entre os pediatras que parecem ser os principais responsáveis pelo manejo de pacientes coréicos em nosso meio nos levou a explorar associações entre as variáveis coletadas para levantar hipóteses que expliquem esta observação.

Encontramos associação entre o uso irregular de profilaxia secundária para coréia e o tempo de formados dos médicos entrevistados. Médicos formados há menos de 10 anos tendem a prescrever a profilaxia secundária de modo mais irregular.

Talvez, a exemplo do que ocorreu em países desenvolvidos ${ }^{24}$, a redução nos casos de FR ao longo dos anos possa ter diminuído, paralelamente, a ênfase deste tópico no curso médico. Além disso, é provável que a redução da importância da FR em países desenvolvidos tenha levado livros-texto e artigos científicos a enfatizarem menos a importância da profilaxia secundária para pacientes coréicos.

Sobre o tratamento da coréia observamos que $28,9 \%$ dos entrevistados elegeram o haloperidol como única droga para tratamento de coréia em fase aguda (Tabela 1); a segunda droga mais citada como monoterapia foi o corticóide, ainda assim com apenas $2,2 \%$ das respostas. Além desta predominância absoluta do haloperidol como droga única para tratamento da CS, em todos os subgrupos de entrevistados, observamos que entre todos os entrevistados que referiram o uso de mais de uma droga, o haloperidol estava presente na maioria destas associações de drogas.

Nosso estudo não foi capaz de estabelecer uma ordem de preferência de uso de drogas para aqueles entrevistados que referiram uso de mais de uma droga na terapia da coréia. Assim, não podemos afirmar que para estes médicos o haloperidol é a droga de escolha. Não obstante, é notável o fato de que, entre todos os entrevistados que usam mais de uma droga para tratamento da coréia, apenas $1,1 \%$ não citou 0 haloperidol como uma das possibilidades. Na pesquisa feita em prontuários do IPPMG, encontramos o percentual de $43,5 \%$ de citações do haloperidol como única droga usada no tratamento da coréia. Esta constatação corrobora observações retrospectivas prévias que apontam o haloperidol como droga de escolha para o tratamento da CS em nosso meio ${ }^{6}$.

É interessante notar a discrepância existente entre a situação retratada na literatura médica sobre o tratamento farmacológico em CS e a situação encontrada por este estudo entre médicos que referem tratar a coréia. Enquanto na primeira encontramos a incerteza e a multiplicidade de condutas, na segunda observamos uma espécie de padronização tácita.

Algumas explicações podem ser aventadas. O haloperidol é referido como tratamento para coréia em artigos científicos há muitos anos, pelo menos desde a década de $1960^{25}$. Nesta época, a FR preocupava a todos, inclusive países desenvolvidos. Anos mais tarde, porém, houve diminuição significativa dos casos de FR nos países desenvolvidos, levando a estiagem de artigos sobre o tema. Assim, a noção pode ter se perpetuado até os dias de hoje.

Entretanto, é verdade que o haloperidol não foi a única droga usada para tratar coréia na década de sessenta, pois que o uso do corticóide também data desta época. O fato dos primeiros trabalhos com o corticóide preconizarem doses tão altas do hormônio quanto $6,6 \mathrm{mg} / \mathrm{kg} / \mathrm{dia}^{26}$ pode ter ocasionado aparecimento de efeitos colaterais indesejados na prática diária, contribuindo para seu uso mais restrito entre os médicos.

Outra possibilidade aventada é a de que o haloperidol teria um duplo efeito para o controle dos movimentos por ser um antipsicótico. Ou seja, o haloperidol, além de reduzir a intensidade dos movimentos coréicos, também produziria melhora nestes sintomas comportamentais que são queixas freqüentes entre os pacientes. 
Assim, os médicos tenderiam a prescrever o haloperidol em detrimento de outras medicações como, por exemplo, o corticóide, carbamazepina e ácido valpróico, cujo efeito sobre comportamento seria bem menor ou inexistente.

Além disso, acreditamos que o fato da Sociedade Brasileira de Pediatria e a Sociedade de Pediatria do Estado do Rio de Janeiro citarem apenas o haloperidol como tratamento farmacológico para coréia pode influenciar, principalmente junto aos pediatras gerais, a prescrição em massa desta medicação para pacientes coréicos ${ }^{27,28}$.

Apesar da uniformização encontrada entre os entrevistados, observamos certas particularidades entre determinados subgrupos de nossa amostra que devem ser ressaltadas, pois, de certa forma, ajudam a elucidar a preferência geral pelo haloperidol.

Análise feita neste estudo comparou os grupos de pediatras dos centros de referência e de fora dos centros de referência. Além da maior predileção, já referida, pelo haloperidol no grupo de fora dos centros de referência, observamos que o uso de corticóide para fase aguda é maior entre os pediatras dos centros de referência (Tabela 2 ).

Considerando-se os pediatras de emergência como um todo e os demais pediatras especialistas, notamos uma maior tendência dos primeiros em prescrever o haloperidol como única droga para tratamento de coréia. O uso de ácido valpróico para fase aguda da coréia também foi maior entre os pediatras dentro dos centros de referência (Tabela 2). Com base neste achado nos indagamos se os pediatras de fora dos centros de referência sabem que o ácido valpróico é uma opção terapêutica para CS.

$\mathrm{Na}$ literatura os trabalhos mais antigos ${ }^{29,30}$, ao indicar uma medicação para coréia, aconselham o médico a ponderar sobre os possíveis efeitos colaterais da medicação frente a uma coréia de intensidade menor. Alguns trabalhos estimam que coréias graves correspondam apenas a $16 \%$ do tota ${ }^{30}$. Ou seja, coréia não é, com base nos melhores dados disponíveis, indicação absoluta de tratamento farmacológico.

Entretanto, quase dois terços dos entrevistados referem tratar farmacologicamente a coréia independentemente de sua gravidade (Tabela 1 ). $O$ alto percentual observado de indicação de tratamento farmacológico para coréia foi corroborada pela análise dos prontuários pesquisados, onde observamos que $100 \%$ deles continham alguma medicação para tratamento da coréia. Acreditamos que a alta freqüência de prescrição de drogas seja, em parte, devida à dificuldade de se determinar a gravidade da coréia de modo mais objetivo, graças ao escasso uso de escala de gravidade.

A pequena freqüência do uso de escala de gravidade e o desconhecimento pela maioria de sua existência (Tabela 1), admitem algumas explicações. Particularmente em emergências médicas, onde há grande demanda de pacientes, o pouco tempo disponível para o atendimento de cada caso justificaria a simplificação do atendimento e o não uso de escalas.

Adicionalmente, a escala de gravidade específica foi publicada em revista especializada em distúrbios do movimento ${ }^{20}$, de língua inglesa, sem qualquer difusão entre pediatras ou especialistas que não da área neurológica. Confirmando tal informação nos questionários, dos prontuários pesquisados, apenas 8,7\% apresentavam referência a algum tipo de gradação para coréia. Apesar das escalas de gravidade, ao nosso ver, não conseguirem excluir completamente a subjetividade na classificação da coréia, elas são, sem dúvida, um avanço, uniformizando esta observação. Em hospitais públicos onde os pacientes são vistos por vários profissionais, o uso de escalas poderia reduzir o tempo de tratamento, por proporcionar mais adequada monitorização de melhora dos sintomas, e a própria indicação de tratamento farmacológico, pois pacientes com coréia leve, em princípio, não necessitariam de tratamento.

Em conclusão, apesar de algumas limitações, em particular quanto à amostra de conveniência obtida entre pediatras de fora dos centros de referencia para o atendimento da FR, verificamos que a coréia reumática é tratada farmacologicamente, principalmente com haloperidol independentemente de sua gravidade, e que ocorre prescrição inadequada de profilaxia secundária. Estes resultados sugerem que ensaios multicêntricos devam ser desenvolvidos para avaliar as drogas disponíveis, com maior divulgação de resultados em revistas e encontros científicos nacionais. Que se revejam, à luz das evidências, periodicamente as recomendações de tratamento farmacológico feitas pelas Sociedades Médicas e se incluam no conteúdo programático oferecido na graduação médica. A divulgação de escalas de gravidade para coréia deve ser maior entre pediatras especialistas e não especialistas como uma forma de incentivar a uniformização do acompanhamento destes pacientes e racionalizar a indicação de tratamento farmacológico. Acreditamos ser importante a realização de 
estudos que comprovem a hipótese levantada sobre o uso inadequado de penicilina benzatina entre pacientes coréicos, levando em consideração o papel do médico em indicar adequadamente esta medicação.

\section{REFERÊNCIAS}

1. Special Writing group of the Committee on Rheumatic Fever, Endocarditis and Kawasaki disease of the Council on Cardiovascular Disease in the young of the American Heart Association. Guidelines for the diagnosis of rheumatic fever. JAMA 1992;268:2069-2073.

2. Oliveira SKF, Azevedo ECL. Reumatologia pediátrica. Rio de Janeiro: Revinter, 2000.

3. Jordan LC, Singer HS. Sydenham chorea in children. Curr Treat Options 2003;5:283-290.

4. Hilário MOE, Terreri MTSLRA. Rheumatic fever and post-streptococcal arthritis. Best Pract Res Clin Rheumatol 2002;16:481-494.

5. Jankovic J, Tolosa E. Parkinson's disease and movment disorders. Baltimore: Williams \& Wilkins, 1998.

6. Araújo APQC, Pádua PAB, Maia HS. Management of rheumatic chorea: an observacional study. Arq Neuropsiquiatr 2002;60:231-233.

7. Diament A, Cypel S. Neurologia Infantil. São Paulo: Atheneu, 1984.

8. Carapetis JR, Currie BJ. Rheumatic chorea in northern Australia: a clinical and epidemiological study. Arch Dis Child 1999;80:353-358.

9. Swedo SE. Sydenham's chorea: a model for childhood autoimmune neuropsychiatric disorders. JAMA 1994;272:1788-1791.

10. Terreri MTRA, Roja SC, Len CA, Faustino PC, Roberto AM, Hilário MOE. Sydenham chorea: clinical and evolutive characteristics. Sao Paulo Med J 2002;120:16-19.

11. Bonthinus DJ, Karacay B. Sydenham's chorea: not gone and not forgotten. Semin Pediatr Neurol 2003;10:11-19.

12. Lubetzki KI, Brand A, Steiner I. Recurrence of Sydenham chorea. Arch Neurol 2004;61:1261-1264.

13. Robertson WC, Smith CD. Sydenham chorea in the age of MRI: a case report and review. Pediatr Neurol 2002;27:65-67.

14. Ayoub EM. Resurgence of rheumatic fever in the United States: the changing picture of a preventable illness. Postgrad Med 1992;92:133142.
15. Garvey AM, Swedo SE. Sydenham's chorea clinical and therapeutic update. Adv Exp Med Biol 1997;418:115-120.

16. Edwards MJ, Dale RC, Church AJ, Giovannoni G, Bhatia KP. A dystonic syndrome associate with anti-basal ganglia. J Neurol Neurosurg Psychiatry 2004;75:914-916.

17. Harrison NA, Church A, Nisbet A, Rudge P, Giovannoni G. Late recurrences of Sydenham's chorea are not associated with anti-basal ganglia antibodies. J Neurol Neurosurg Psychiatry 2004;75:1478-1479.

18. Colony SH, Malamud N. Sydenham's chorea: a clinicopathologic study. Neurology 1956;6:672-676.

19. Dornaus C, Jacob C, Kiss MH, Oselka GW. Tratamento da coréia de Sydenham com haloperidol. Rev Paul Med 1984;102:81-83.

20. Cardoso F, Eduardo C, Silva AP, Mota CC. Chorea in fifty consecutive patients with rheumatic fever. Mov Disord 1997;12:701-703.

21. Katzung BG. Farmacologia Básica \& Clínica. Rio de Janeiro: Guanabara Koogan, 1998.

22. Dajani A, Taubert K, Ferrieri P, Peter G, Shulman S \& Committe on Rheumatic Fever, Endocarditis, and Kawasaki Disease of the Concil on Cardiovascular Disease in the Young, the American Heart Association. Treatment of acute streptococcal pharyngitis and prevention of rheumatic fever: a statement for health professionals. Pediatrics 1995;96:758764.

23. Behrman RE, Kligman RM, Jenson HB. Nelson textbook of pediatrics. Philadelphia: Saunders, 2004.

24. Harel L. Rheumatic fever: a never-ending story? Isr Med Assoc J 2000;2: 480-481.

25. Axley J, Mallinckrodt E. Rheumatic chorea controlled with haloperidol. J Pediatr 1972;81:1216-1217.

26. Siqueira LC, Oliveira A. Tratamento da coréia de Sydenham pelos esteróides a curto prazo. Bol Inst Puer Univ Brasil 1965;22:113-116.

27. Sociedade Brasileira de Pediatria. Educação médica continuada em Febre reumática, elaboração de julho de 2002. Disponível em:<http:/ / www.sbp.com.br>. Acessado em: 08 mar.2006.

28. Sociedade de Pediatria do Estado do Rio de Janeiro. Disponível em:<http:/ /www.soperj.org.br> . Acessado em: 08 mar.2006.

29. Shields DW, Bray PF. A danger of haloperidol therapy in children. J Pediatr 1976;88:301-303.

30. Alvarez LA, Novak G. Valproic acid in the treatment of Sydenham chorea. Pediatr Neurol 1985;1:317-319. 